BIO Web of Conferences 4, 00012 (2015)

DOI: $10.1051 /$ bioconf $/ 20150400012$

(C) Owned by the authors, published by EDP Sciences, 2015

\title{
Origins of life: From the mineral to the biochemical world
}

\author{
Jean-François Lambert ${ }^{1,2}$ \\ ${ }^{1}$ Sorbonne Universités, UPMC Univ Paris 06, UMR 7609, LRS, Case Courrier 178, \\ 94200 Ivry-sur-Seine, France \\ ${ }^{2}$ CNRS, UMR 7609, LRS, Case Courrier 178, 94200 Ivry-sur-Seine, France
}

\begin{abstract}
In this brief review, we summarize the most influential scenarios in origins-oflife studies that have resorted to minerals surfaces. The influence of the pre-existing mineral world on the organization of matter towards the living state may have manifested at several steps in the rise towards higher complexity. Small biomolecules such as amino acids formed on mineral surfaces of interstellar dust, meteorites or comets prior to their delivery to the primordial Earth. Mineral surfaces are known to promote the polymerization of these small molecules to the corresponding biopolymers (e.g. amino acids to proteins), and they may have oriented this polymerization to the formation of specific structures. Crucial steps of protometabolism could have originated on the surface of sulfide minerals. The most daring view of the prebiotic role of minerals holds that some of them may have carried genetic information subject to darwinian selection prior to the rise of nucleic acids, today's genetic material. Finally, porous structures ranging from the nanometer to the micrometer probably helped protect the first proto-organisms from dilution and other damage. Future studies are expected to see progress towards molecular-level characterization, and to start accounting for the complexity of the prebiotic environment.
\end{abstract}

\section{Prebiotic studies: The rise to complexity}

The question of the origins of life is one of the most challenging facing science today. It has been the object of continuous effort from a dedicated community of scientists from diverse horizons for almost a century, including some of the brightest in their fields. It would be presumptuous to try and summarize such a vast topic in a few pages; the present contribution is more modestly dedicated to assessing some scenarios that specifically address the possible role of a pre-existing, well-structured mineral world as a "scaffold" to organize carbon-based molecules into the complex systems that we now see thriving around us.

When dealing with the complexity of life, the scientist's first reflex is to reduce it to ever simpler levels of organization following the principles of Cartesian analysis (Fig. 1).

Thus, the organisms that we meet in everyday life are composed of cells. The latter comprise several functional systems, which may be localized in organelles in the case of eukaryotes (e.g. respiration in mitochondria). In turn the functioning of these systems rests on specialized biomolecules such as proteins (enzymes, etc.) and nucleic acids, which are themselves polymers of simpler units such as

This is an Open Access article distributed under the terms of the Creative Commons Attribution License 4.0, which permits unrestricted use, distribution, and reproduction in any medium, provided the original work is properly cited. 


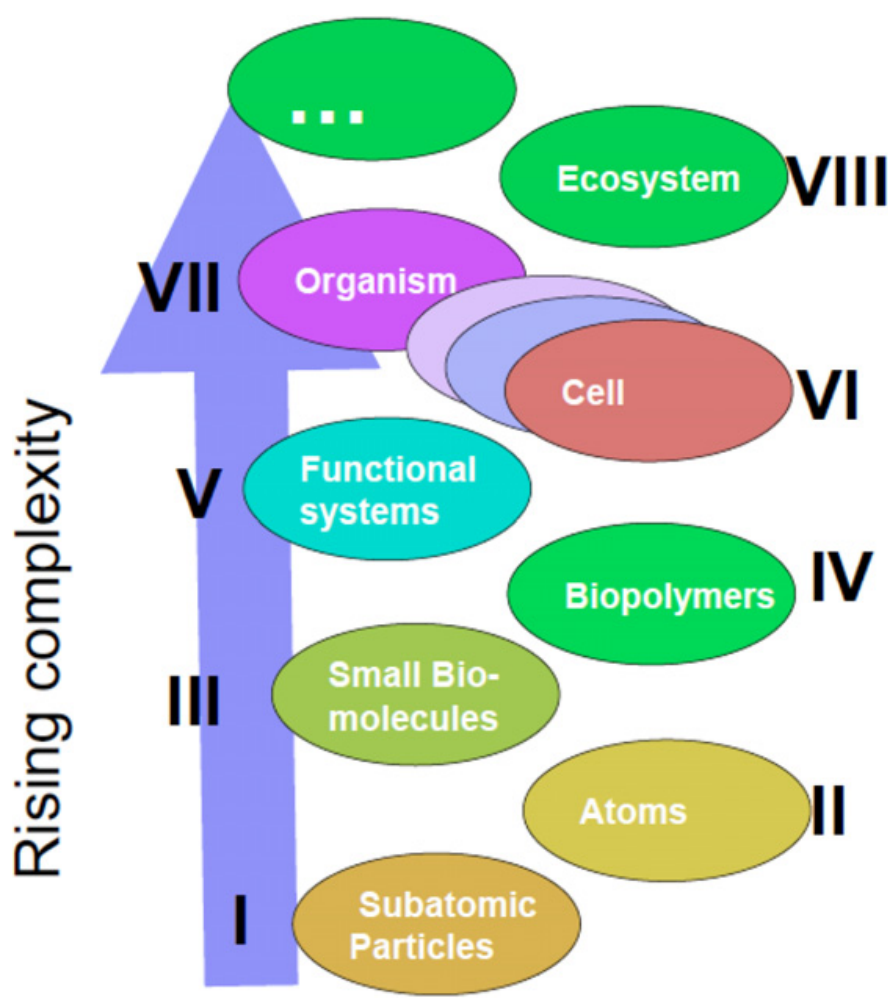

Figure 1. Successive levels of organization existing in the living world.

amino acids and nucleotides; finally, these small molecules are composed of atoms and elementary particles.

It is tempting to believe that this intricate complexity arose stepwise, following the same sequence in reverse order. This approach is certainly a typical application of Descartes' method, as it attempts to reduce a problem of daunting complexity to a sequence of manageable steps. More holistic approaches are possible and maybe intellectually more challenging $[1,2]$. However, in the present article we will use the picture of a stepwise rise to complexity, as it constitutes a practically useful device to put into perspective several problems related to the origin of life.

Once we reach complexity level VI, that of the cell, we have a satisfactory theory to describe further happenings, the modern evolutionary synthesis - LUCA, the last universal common ancestor of all living beings, is supposed to have been similar in complexity to a present day cell. Some of the preceding steps however are very challenging to understand.

\section{Origin of small biomolecules}

\subsection{Biomolecules have emerged in many cosmic laboratories}

How the constituent atoms of life, including carbon, were created as the Universe came of age is neatly explained by the astrophysicists who unraveled the process of stellar evolution. This is a fascinating story, but one that predates both the mineral and the biological worlds. As the newly created elements were spewed out of a dying star, and later when molecular clouds collapsed into a proplyd during the formation of a star of the next generation, they combined according to the laws of chemistry, in 


\section{ORIGINS}

varying conditions of temperature and pressure. When the process of solar system formation was almost completed, elements had been combined and shuffled according to the volatility of the resulting species. Reduced transition metals, and various combinations with silicon, aluminum, magnesium, oxygen and sulfur, formed the bulk of the inner rocky planets. Lighter elements that would become crucial to life, especially carbon and nitrogen, had a stronger tendency to form small volatile molecules such as methane $\left(\mathrm{CH}_{4}\right)$ or ammonia $\left(\mathrm{NH}_{3}\right)$, and these were relegated to more distant locations beyond the "ice line", the gas giants, comets and some asteroids. This was also true of the most important molecule for life - water, $\mathrm{H}_{2} \mathrm{O}$.

Each world - the Earth, Mars, Jupiter... each comet and asteroid - was a chemical laboratory with its own set of parameters. Some were cold, some were hot, some had an atmosphere, some were rich in carbon... These divergent worlds gave rise to diverse organic chemistries that are the object of active research by astrochemists [3]. While some of them remain alien to us and could constitute independent experiments in organic complexity (e.g. Titan [4]), what happened on comets and asteroids is certainly relevant to the history of life on Earth since a number of them crashed on Earth during the "late heavy bombardment" period, delivering their contents of organics and/or water to our planet [5]. Direct observation of planetary systems in the making reveals that molecules with rather complex carbon skeletons are indeed formed at this stage, and also molecules that contain functions important for life such as amines and carboxylic acids. The evidence is still inconclusive regarding the presence in foreign systems of the more typical small biomolecules, e.g. amino acids, even the simplest one, glycine. Closer to us, however, the analysis of meteorites has shown that carbonaceous chondrites do contain a variety of amino acids, as well as (but in smaller amounts) the nucleic bases that are a crucial component of nucleotides, the second most important family of biological monomers ([6]; perhaps sugar precursors were formed too [7]). The structure of amino acids and nucleotides is recalled in Fig. 2. They may also have been present in comets; at the time of writing these lines, the Philae lander had just arrived on comet Churyumov-Gerasimenko in an attempt to determine how complex its organic molecules might be, and the success of this mission was still unknown.

So more or less complicated organic molecules were delivered on Earth, there to continue their evolution in chemically different environments. Small biomolecules were either already present, or they were formed by Earth-based chemistry. The early experiments of Miller and Urey [8], certainly the bestknown in prebiotic chemistry, were meant to show that electrochemical activation of simple components in the early Earth atmosphere could produce biomolecules (amino acids again). Even though their assumptions on atmospheric composition were later challenged, this is not considered to be a basic flaw. Between atmospheric/cometary and Earth conditions, it is highly likely that the most typical small biomolecules were synthesized in significant amounts by the rules of everyday chemistry - i.e. stage III was reached without too much difficulty.

\subsection{The role of minerals in small biomolecules syntheses}

Did minerals play a role in this step? Most probably, they intervened as catalysts - through a temporary interaction with small precursor molecules, they opened up easy reaction pathways directing them, for instance, towards amino acid formation: in this way, a reaction that was a mere thermodynamic possibility was actualized within a reasonable time lapse - rather than some alternative reactions that were "useless" for life emergence. This capability of mineral surfaces is well-known and largely used in the chemical industry. Of course, we have to ask the question of what mineral phases were actually available at the time, and whether they had any catalytically interesting properties. A rather recent development in geological science is the realization that the mineral world has had an evolution in terms of complexity and diversity, that may rival those of the biological world [9]. The mineral species existing on the primeval Earth, and all the more on little-evolved asteroids, were fewer than today. But they included materials with well-known catalytic properties; indeed, a speck of dust collected 
<smiles>[2H][C@@H](N)C(=O)O</smiles><smiles>[R][C@]([2H])(NC(=O)[C@@H]([2H])NC(=O)[CH]C)C(=O)NCC</smiles><smiles>Nc1ncnc2c1ncn2[C@@H]1O[C@H](COP(=O)([O-])OP(=O)([O-])OP(=O)([O-])[O-])[C@@H](O)[C@H](O)[C@H]1O</smiles><smiles>O=P([O-])([O-])OP(=O)([O-])OP(=O)([O-])[O-]</smiles><smiles>Nc1ncnc2[nH]cnc12</smiles><smiles>OC[C@H]1O[C@H](O)[C@@H](O)[C@H](O)[C@H]1O</smiles><smiles>CCOP(=O)([O-])OC[C@H](O)[C@@H]1O[C@H](n2cnc3c(N)ncnc32)[C@H](O)[C@H]1OP(=O)([O-])OCC</smiles>

Figure 2. Top: chemical structure of L-amino acids, and of their polymers (peptides); bottom: chemical structure of a nucleotide (adenosine triphosphate), of its more elementary constituents (ribose, adenine, triphosphate ion), and of its polymer (RNA).

from comet Wild-2 by the Stardust mission consists in nanometric $\mathrm{Ni}$ and $\mathrm{Fe}$ particles supported on a silicate matrix ([10], see Fig. 3) - a formulation that looks oddly familiar to specialists in heterogeneous catalysis, and would be able to catalyze many industrially relevant organic reactions such as hydrogenation/dehydrogenation, or maybe Fischer-Tropsch synthesis [11]. The latter actually refers to an industrial process that was initially devised to produce oil substitutes from a mixture of $\mathrm{CO}$ and $\mathrm{H}_{2}$; it is highly relevant from a prebiotic point of view because the crucial step induces the formation of $\mathrm{C}-\mathrm{C}$ bonds from one-carbon precursors, that is, the buildup of a carbon skeleton from one-carbon molecules.

Extant data on heterogeneous catalysis by mineral phases usually refer to industrial processes that must be very fast (in the order of seconds to minutes) in order to be efficient. Therefore, they are carried 


\section{ORIGINS}

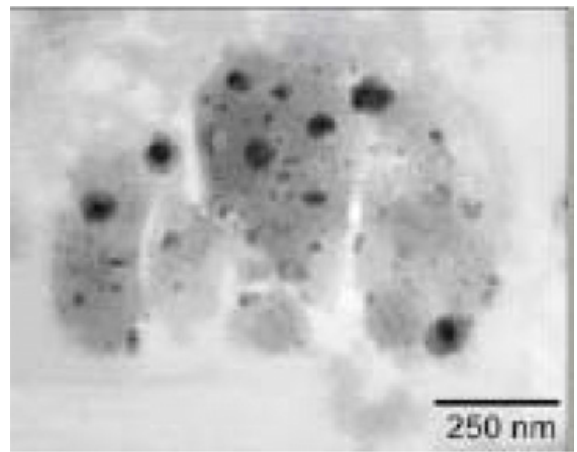

Figure 3. A famous speck of dust: a particle ejected from a comet and collected by the Stardust mission. The dark inclusions are Fe and Ni metal and metal sulfide nanoparticles, the gray matrix is a silicate support.

out at rather high temperatures, unlikely to have been reached on Earth. However, it is not difficult to extrapolate reaction rates to lower temperatures, and many catalytic reactions remain fast enough in more lenient conditions, at least on a geological time scale.

To summarize, it is highly likely that the preexistence of a complex mineral world was a necessary condition in the emergence of even the simplest biomolecules.

\subsection{The question of chirality selection}

One of the most striking features of biomolecules is their chirality. Between the two mirror images that exist for most amino acids for example, life uses only one (the $\mathrm{L}$ form, or L enantiomer), with zero tolerance for the other (the D form). In contrast, known prebiotic pathways such as Miller-type experiments only produce racemic mixtures, which contain exactly as much of the $\mathrm{D}$ as of the $\mathrm{L}$ form. At one time in the prehistory of life a selection of the $\mathrm{L}$ form must have happened, and how this came about is one of the most enduring problems of prebiotic chemistry; a whole book has been devoted to the many hypotheses put forward to explain chirality selection [12].

One of these involves enantioselective adsorption on crystal faces. Hazen et al. [13] have investigated the adsorption of the two forms of an amino acid (aspartic acid) on well-defined faces of calcite crystals. These faces themselves already possess the property of chirality, i.e. the arrangement of atoms exposed on the surface is not superimposable to its mirror image. Perhaps not surprisingly, they were found to exhibit preferences for one of the enantiomers of aspartic acid: while the (21 $\overline{3} 1)$ faces adsorbed a little more of the $\mathrm{D}$ than the $\mathrm{L}$ form, the reverse was true for their mirror image, namely the $(\overline{3} \overline{1} 21)$ faces. The difference was not great, about $1 \%$; but there are known mechanisms that allow an amplification of small chirality differences (see Chapter 10 in ref. [12] for a review). This is an instance of information transfer from the mineral to the inorganic world as the chirality preexisted in the mineral, as it does in others such as quartz $[14,15]$. It has been objected that the two enantiomorphic forms of chiral surfaces are present in equal amounts in most settings [16] which makes it hard to understand how differential adsorption could have resulted in significant concentration of an amino acid enantiomer.

\section{Origin of biopolymers}

\subsection{The problem with polymerization}

Suppose that step 2, the prebiotic synthesis of small biomolecules, has already been largely completed. Here we are, then, some time in the middle of the archean eon, when devastating blows by celestial 
bolides have become less threatening and primordial chemistry has resulted in the formation of many organic molecules including some building blocks of life, so that the oceans consist in a warm and dilute "primordial soup". People realized that this was a most likely outcome of the laws of nature in the early 1950 's, and the natural reaction was to conclude that the mystery of the origins had more or less been solved. This was grossly overoptimistic. The contents of a living cell do not resemble a "soup" - they are highly organized. Let us consider the next step in the rise towards complexity, i.e. the formation of biopolymers (stage IV). It may not seem difficult to combine monomers into a string-like structure, but chemical thermodynamics predicts otherwise: polymerization should not be favored at all in aqueous solution, as several authors have underlined $[17,18]$.

To be more precise, let us suppose that our primordial soup is highly concentrated in monomeric amino acids, e.g. it contains 0.5 mole of glycine per liter, which seems very high. Suppose further that we are interested in the formation of a 15-residue polypeptide; this figure is chosen because polypeptides of that length are sometimes complex enough to exhibit interesting properties such as self-duplication. This requires the formation of 14 amide bonds between a $-\mathrm{COOH}$ and a $-\mathrm{NH}_{2}$ residue (see Fig. 2), and each one is thermodynamically uphill: technically, the free enthalpy of reaction is positive. It is easy to calculate the equilibrium concentration of the requested polymer in the soup. It is so low that, in order to encounter a single molecule of polymer, one would have to scan through $10^{50}$ tons of solution; to put this result into perspective, the total mass of the Earth is only $6.10^{21}$ tons. Now this is a simplified calculation as it completely neglects the fact that a functional peptide is not obtained by a random polymerization, but must contain specific residues in a well-defined order. Factoring this requirement into the calculations further decreases the favorable odds, by many orders of magnitude. It will not do to say that even unlikely events will finally happen given enough time. There has simply not been enough time (by far) since the origin of the Earth for a single molecule of useful peptide to have formed by random combinations.

\subsection{Biopolymerization and mineral surfaces}

Therefore, polymerization must have taken place in a different way. In an influential book published in 1951 [19], Bernal suggested that polymerization may have occurred between biomolecules adsorbed on a mineral surface. The original argument was that adsorption locally increases the effective concentration, which always favors the association of molecules. While the effect is probably insufficient, it remains that thermodynamical parameters might be different in the adsorbed state from the free solution, because of different interactions with the surface between the monomers and the polymers, possibly making the polymerization thermodynamically favorable.

Attempts at testing Bernal's hypothesis have been carried out since the 1970's. Lahav [20, 21] had the idea of studying the reactivity of non-activated amino acids in fluctuating environments. A solution containing the monomers was contacted with clay minerals, and the system was dried at moderate temperatures; then it was wetted again, and the cycle was repeated several times, with the aim of mimicking climate changes that could have happened in real environments (a succession of rains and dry periods). These and other teams' investigations [22, 23] definitely observed the formation of oligopeptides, up to the decamer in some cases.

It took a long time until we began to understand why these polymerization procedures were successful. In fact, the mineral surfaces have little influence, if any, on the polymerization thermodynamics [24]. What makes polymerization possible is the coupling with macroscopic transformations in which the system regular passes through a state of low water activity [25]. The reaction of peptide bond formation is a condensation, i.e. it produces one water molecule for each bond formed. A well-known rule of thermochemistry is Le Châtelier's principle: removing the products of any reaction drives the equilibrium to the right. Drying means removing water, and therefore favors condensation. Thus, the surfaces tested have in fact at most a minor influence on reaction 


\section{ORIGINS}

thermodynamics; however, they may still play an important catalytic role as their interaction with amino acids allows them to condense at a much lower temperature than they would e.g. in the bulk. Not all surfaces are equal from this point of view, and for instance amino acids supported on silica are observed to polymerize at significantly lower temperatures than on clay minerals [26], even if both systems are "equally dry". An important notion that emerges here is that of catalytic sites, special arrangements of atoms on the mineral surface that are required to activate the reagents in a well-defined way. In other words, a mineral surface is not a mere geometrical plane, but a chemical system that needs to be studied at the molecular level, just as the biomolecules that adsorb on it.

In the same way as amino acids, nucleotides were observed to polymerize catalytically on a clay surface, although the corresponding studies used activated monomers (phosphorimidazoles) that are prebiotically unrealistic and eschew the thermodynamic problem [27].

\subsection{Polymerization selectivity and organization}

Scenarios involving adsorption and thermal reaction on mineral surfaces may provide a path to biopolymers, either alone or in combination with natural activating molecules [28]. However, as already alluded to, it is not enough to make peptides from amino acids, or nucleic acids from nucleotides. Random polymerization from a solution containing several types of monomers would cause the accumulation of a huge variety of polymers, most of which would be completely useless for further organization. To avoid being mired down in hopeless complexity, some process must allow to orient the reaction towards a limited number of products, by selecting the monomers that are allowed to react, and/or by imposing special sequences during polymerization itself. In other words, we must answer the different but connected questions of adsorption selectivity and polymerization selectivity.

Adsorption selectivities certainly exist at least for amino acids, as proved by the use of mineral phases such as silica for chromatographic separation [29]. They have been very little studied in the context of prebiotic chemistry. Nevertheless, it is quite obvious that some minerals can discriminate between amino acids on the basis of their electric charge in the solution. For instance, a clay mineral is composed of layers bearing a negative charge (of substitutional origin), and it will specifically intercalate between its layers those amino acids that have a positive charge at moderate $\mathrm{pH}$ (the charge on the molecule depends on acid-base equilibriums, and therefore on solution $\mathrm{pH}$ ). Thus, in a solution containing negatively charged glutamate (Glu) and positively charged arginine (Arg), montmorillonite overwhelmingly adsorbs arginine [30], while the exact opposite is true for adsorption on hydrotalcite, another mineral that bears negative substitutional charge.

As regards polymerization selectivities, there are some tantalizing clues in the literature showing that dipeptides [31] and tripeptides [32] are not formed randomly on some mineral surfaces but some sequences are favored over others. It was also claimed that copolymerization of glutamic acid and glycine on alumina [33] resulted in long alternate (Gly-Glu) polymers, and that copolymerization of glutamine and glycine on titania yielded alternate (Gly-Gln) $)_{n}$ [34]. In the same way, mixed species with equal numbers of each amino acid were observed preferentially upon thermal activation of (Glu+Arg) on montmorillonite clay [30], but this preference for alternate polymers may be due as much to the properties of the amino acids themselves as to the influence of the surface [35]. If we turn now to the other large family of biomolecules that have been polymerized on minerals, namely nucleotides, we see that according to the uncontested specialists of the field, the insertion of nucleotides in a growing polymer is not random but follows a precise order of preference: $\mathrm{A}>\mathrm{G}>\mathrm{C}>\mathrm{U}$ [36].

Finally, very recent results regarding polyglycine formed on titania indicated that the oligomers interacting with the titania surface seem to have a well-defined secondary structure containing both $\alpha$-helices and $\beta$-sheets, judging from the shape of their IR bands [37]. This is important since this kind of structural elements play a fundamental role in defining the reactivity of proteins: thus, the functional elements of protein may have already been in place at this step. 


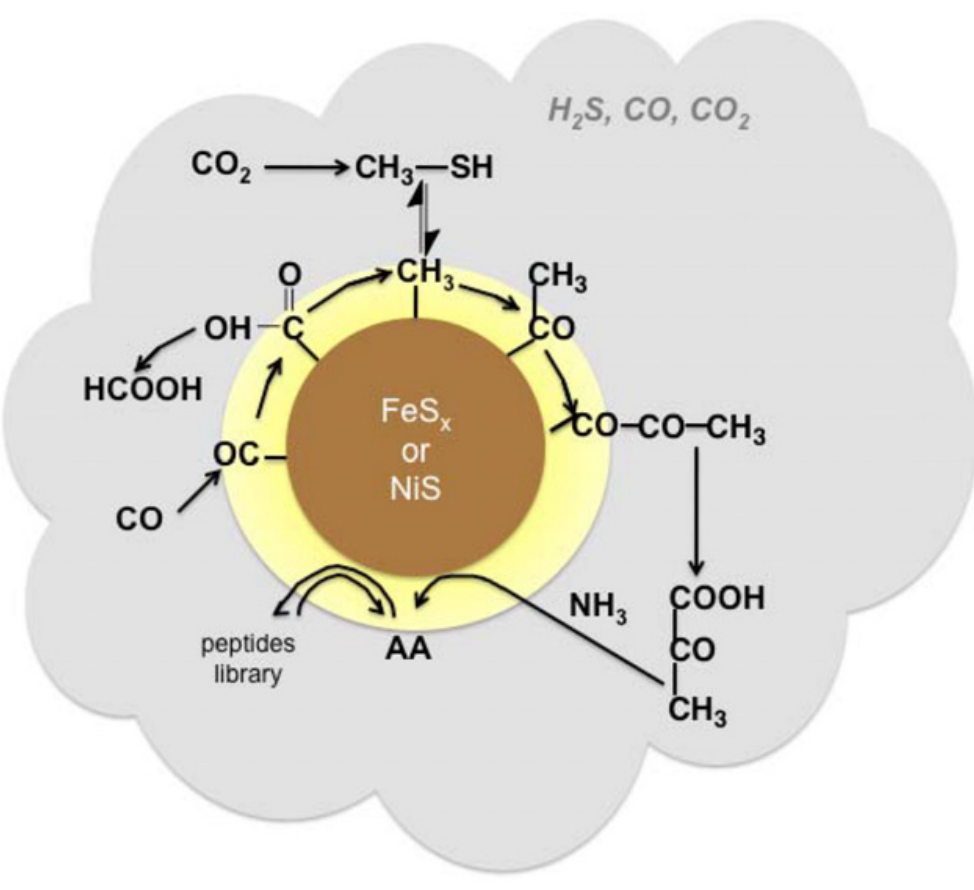

Figure 4. A sketch of the main protometabolic reactions proposed by Wächtershäuser to have occurred at the surface of metal sulfides.

As we can see from this summary, there are promising aspects in surface polymerization scenario, but this domain of research is still in its infancy.

\section{Origin of metabolism}

Going from level IV to level V implies actuating function, typically by harnessing free energy flows in metabolic pathways. In modern cells this is done by exquisitely fine-tuned assemblies of biomacromolecules working in cooperation (think for instance of the arrays of enzymes embedded in mitochondrial membranes involved in respiration). If such a level of complexity needed to be reached before the chemistry of life really started working, things may look rather hopeless. But there might have been different ways to kick-start protometabolism.

At the turn of the 1990's, G. Wächtershäuser caused quite a stir in the origins of life community by constructing more and more precise models of protometabolic pathways that might have occurred at the surface of metal sulfide minerals [38-41]. The free energy source would have been the formation of disulfide ions from sulfides, favored by the precipitation of pyrite $\left(\mathrm{FeS}_{2}\right.$ [39]), and the electrons given up in this transformation (technically, a redox half-reaction) would have been used to reduce $\mathrm{CO}_{2}$ to organic matter. A partial view of the resulting transformations is shown in Fig. 4: the reader can see that they would have proceeded as far as amino acids (AA), or even polypeptides, starting from simple materials which were likely present in the primordial atmosphere such as $\mathrm{CO}, \mathrm{CO}_{2}$ and $\mathrm{H}_{2} \mathrm{~S}$.

This was an impressive intellectual construction, and a timely one as more and more evidence at the time pointed to a "sulfur world" stage in the appearance of life, involving the kind of chemistry that might still be occurring today in submarine black smokers - a place of high temperatures and pressures, strong gradients, and sulfur mineral precipitation. However it remains largely untested experimentally, 


\section{ORIGINS}

although some steps have been partly verified - the fixation of CO to increase organic chain length [42], and the (limited) polymerization of amino acids [43].

A recent development in mineral-based protometabolism was inspired by the fast development of a new energy conversion device, namely, fuel cells. It was shown by Barge, Russell et al. that freeenergy-rich polyphosphates could have been synthesized as a consequence of the existence of $\mathrm{pH}$ gradients across a mineral membrane, such as might exist in black smokers [44]. In such experiments, a macroscopic or "mesoscopic" scale inhomogeneity in the distribution of a chemical component (the protons that define the $\mathrm{pH}$ value) is used as a temporary store of free energy, and upon release of the gradient part of the latter is converted into chemical energy (that of the P-O-P links in polyphosphates). The basic description of this process bears a close similarity to what happens in mitochondria, which also temporarily store energy as a $\mathrm{pH}$ gradient, except that the membrane is now inorganic. The molecular details of the energy conversion process are unknown - but it works. Yet at the same time it shows the limits of our imagination and technical capabilities: the fuel-cell model of protometabolism emerged only after fuel cells became an object of wide industrial and scientific interest.

\section{Clays as living beings and the genetic takeover}

In most people's view, the two main defining functions of life are metabolism (energy transformation) and information storage (today involving DNA and RNA). We have seen that mineral surfaces have helped in the emergence of metabolism; it is logical to wonder if they had anything to do with the preservation and copying of chemical information.

In the 1970's and 80's Graham Cairns-Smith brought a radical approach to the question in several papers and books [45-47]. He actually suggested that some purely mineral systems, and more especially clay minerals, might have been counted as the first living systems, since they would have exhibited some of the basic features of life: their outer properties (phenotype) would have been the consequence of chemical information stored in their lattice in an orderly way (genotype): they would have grown, "reproduced" vegetatively while conserving the defining information, and even been subject to a form of natural selection. Some phenotypes would have been more efficient at aggregating matter than others, and thus they would have been favored in a Darwinian struggle for resources, the corresponding genotype being reproduced in greater numbers than its competitors. Maybe the idea originated from the known fact that some crystalline defects are observed to replicate when the crystal grows; at any rate, it soon became more specific and richer and centered on clay minerals. The latter are fascinating systems in many respects. They are organized in two-dimensional layers stacked on top of each other, sometimes with impressive regularity. In particular, kaolinites exhibit hexagonal platelets piled like the pages of a book - it is not necessary to be particularly imaginative to toy with the idea that they might well carry information. Furthermore the role of clay in the shaping of Man in the traditional creation tales of the Adamic religions may have helped capture the interest of the general public, yet it remains true that clay minerals may bear some information. This information may be of different nature; it may vary continuously, as is the case for the isomorphic substitution rate (for instance, Al for Si substitution), which determines important properties including the ability to swell in water; or it may be discrete, in the case of crystallographic relations between the orientations of successive layers in the stacking. In Figs. 5 and 6, we present a grossly oversimplified view of the ways in which this system may be considered as "living", and later may interact with organic molecules. It does not do justice to Cairns-Smith's thought; it should rather be considered as an idealized cartoon.

In Fig. 6, we see how an originally marginal property of the individual types of clay mineral layers, the preferential adsorption of some members of a chemicals family, could have resulted in the sequential information of the stacking being duplicated in another medium, namely a well-defined sequence of biomolecules. The letter-for-letter translation, or transliteration, that is illustrated here is a highly idealized vision of the process. In reality, the translation must have been imperfect and may 


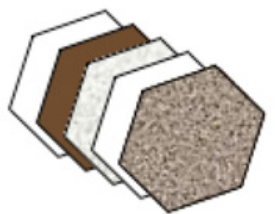

\section{a. «genetic » information}

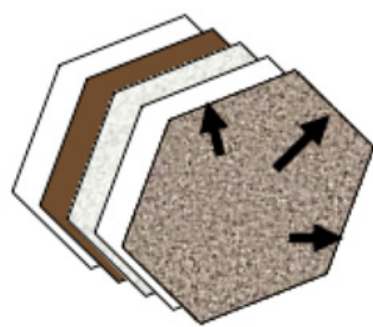

b. growth

Figure 5. a) Illustrates how a stacking of kaolinite layers could be considered as carrying information: crystallographically different layers are represented as different shadings. If they are labeled as type $\mathbf{1}$ to type $\mathbf{4}$, the information string represented here is $\mathbf{1 - 2 - 3 - 1 - 4}$ (note that in practice, there is no reason to envision exactly four distinct types of layers). Cartoons b) and c) respectively illustrate lateral growth of the stacking, and "reproduction" of the phenotype and its associated genotype by fragmentation.

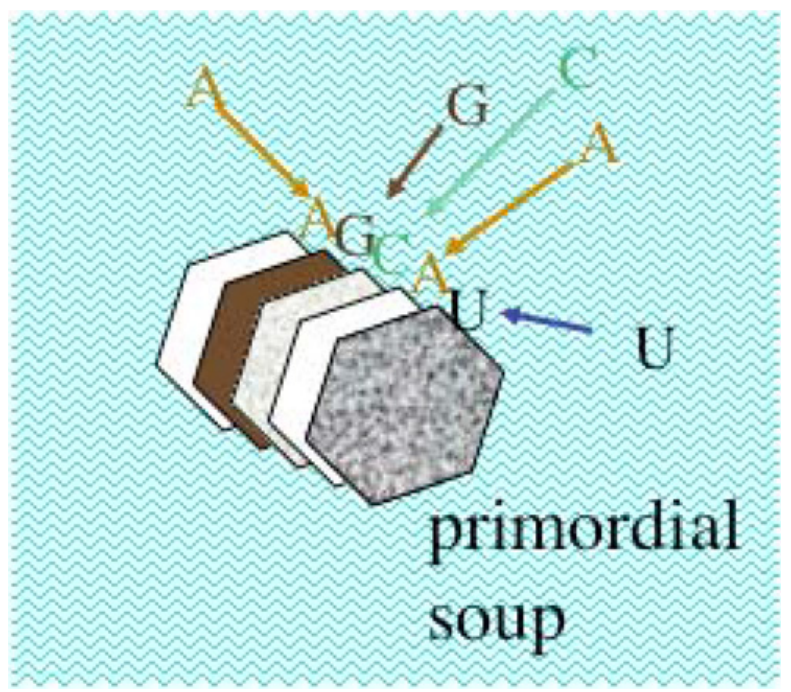

Figure 6. In the first step of genetic takeover, mineral information contained in the clay layers stacking is duplicated, or translated, into biochemical information contained in a string of different biomolecules, as each type of layer preferentially adsorbs one type of biomolecule. Here, for the sake of simplicity, it is supposed that this translation directly involves the familiar nucleosides/nucleotides of RNA. Adenosine is adsorbed on type $\mathbf{1}$ layers, Guanosine on type 2, Cytosine on type 3, Uridine on type 4, so that the mineral-based information string 1-2-3-1-4 is translated into the nucleotide alphabet-based message, A-G-C-A-U. At some point, the message may be stabilized by the formation of phosphate ester linkages (polymerization).

have involved several successive molecular "alphabets". At some point, the mineral phase may also have helped freezing the string of biomolecules by helping them polymerize (see Sect. 3). It is assumed that later the biochemical version of the message may have become autonomous and started replicating on its own; but it could not have originated without its previous interaction with a mineral scaffold.

The idea of a genetic takeover, from the mineral to the biochemical world, is certainly challenging and thought-provoking. Its details remain murky so far; a rather fundamental objection to the scenario 


\section{ORIGINS}

is that a particular sequence of information, that may have some survival or reproduction value in the mineral world, has no reason to be equally adaptative in its biochemical version, e.g. in the RNA world that must have taken over in the history of life. So even if natural selection has promoted the former, it may be indifferent to the latter or even cause its disappearance: it is not clear why there could have been a continuity of information between the two worlds.

There have not been many experimental attempts at confirming the hypotheses in Cairns-Smith's scenario or at implementing it practically. Beyond very general observations that some crystallographic defects do propagate in the process of crystal growth [48], a recent report has more specifically explored the growth of clays of the saponite family [49]. It was found that regions of low, or of high isomorphic substitutions in the clay layer propagated from one layer to the next one in the stacking. This is an interesting fact, but in this case the information is distributed in two dimensions over the surface of the layer. This 2-D information is quite different from the 1-D information that we have envisaged in the previous discussion. 1-D "messages" seem to be more relevant to the emergence of biological information, as it is the kind of information that we find stored in modern genetic systems in the form of nucleic acids - and also the kind of information that we use in human language.

\section{Porous minerals and confinement}

There is one requirement for living systems that does not always find its way into definitions of life, yet is absolutely indispensible. They must stay together rather than progressively diluting into nothingness, the fate that should befall them since it corresponds to the direction of entropy increase. In today's cells (level VI in the organization ladder), they are kept together by encapsulation within vesicles limited by a semi-permeable membrane; in this context, it should be called a largely impermeable membrane.

Vesicles may form spontaneously from solutions containing amphiphilic molecules [50] and the latter, especially fatty acids, are not the most difficult to form in prebiotic conditions. In fact, it has been possible to make membranes and vesicles from organic matter extracted from a meteorite [51]. However, these scenarios for encapsulation work better when the organic matter is rather highly concentrated initially. Since we are precisely trying to explain why the high concentrations were possible in the first place, other explanations have been put forward. In the line of Bernal's original thinking, several authors have remarked that a number of minerals naturally develop a range of porous structures, from the microporosity of zeolites whose dimensions are comparable to the sizes of small molecules (nanometer range), to the micrometer-sized weathering channels that develop in feldspars (technically, they are called macropores) [52]; all of them may have been effective for compartmentalization, and it has even been said that "the first cell wall might have been an internal mineral surface" [53]. The macroporosity of pumices has also been evoked in this respect [54], and a special mention must be made of clay minerals [55], whose expandable interlayers may have provided size-tailored microporosity for a variety of molecules. All of these pores could constitute a rather safe haven for developing prebiotic experiments in organic molecules self-organization, protecting them from dilution, and maybe also from photochemical degradation due to the hard UV photons that reached the surface of the Earth at the time (renewed interest is currently devoted to this possibility in the frame of the exploration of the surface of Mars, where the irradiation is rather similar to what it was on the primordial Earth). In cases where the dimensions of porosity are small enough, it may have oriented organic reactions towards specific outcomes by the effect of molecular confinement. Very little work has been carried out to assess this possibility in the frame of prebiotic chemistry, but we could draw on the vast literature of heterogeneous catalysis for guidelines.

A puzzling question arises if we assume that the first living systems were harbored in the pores of inorganic materials, or between clay layers. In fact this question turns out each time we resort to mineral surfaces to explain a crucial step in prebiotic organization and is only exacerbated here. Namely, if life began on surfaces, how did it detach from them? If a significant interaction with the surface is required, 
it means that the adsorption is favored, all the more when the system size increases from monomers to biopolymers to functional assemblies. There is therefore less and less incentive for the growing and diversifying biological systems to desorb, and they should have stayed forever stuck to the mineral surfaces where they originated. This paradox was clearly formulated by Miller and de Duve [56] and has not yet been satisfactorily resolved. The answer may have involved large-scale cycling in geochemical parameters, which could have induced leaching of the supporting mineral and consequent releasing of the adsorbed organic system (perhaps carrying with it a remnant of the initial mineral phase as a memento of its origins). In an interesting study, Hanczyc et al. [57] showed that montmorillonite clays facilitated the transformation of fatty acids into encapsulating vesicles, a kind of "compartmentalization takeover"; furthermore, small montmorillonite particles were indeed present in some of the resulting vesicles.

\section{Conclusion - the need to investigate molecular diversity on mineral surfaces}

This brief guided tour through "mineral-based" prebiotic scenarios has spanned several decades of prebiotic research. Time and again, researchers including some of the most brilliant minds in the field have underlined that life did not arise in a vacuum, but on a planet that already harbored a vast and diverse mineral world. The emerging molecular assemblies that would become living organisms interacted with this world. Since they were mostly present in dissolved form in the ancient oceans, this interaction took place at the aqueous solution/solid matter interface. The phenomena that occur in this setting have been investigated for a long time by geochemists, soil scientists, and chemists concerned with the industrial applications of these interfaces, for example to heterogeneous catalysis. The vast body of knowledge that has been accumulated on the structure and reactivity of minerals surfaces must now be exploited in the field of prebiotic chemistry. Grand ideas have been proposed concerning the role that minerals may have played in organizing matter in its rise towards life, from simple supports to scaffolds, energy sources or even maybe providers of molecular-level information. Often, experimental verification has been slow to come, or is still waiting. The work that remains to be done is huge. It may yield some decisive insights into the historical path towards life - or not. But at any rate, we will learn something about the possibilities for self-organization of matter.

An idea that we have encountered several times is that of selective adsorption of biomolecules. It certainly happens in some circumstances, but its scope and capability for organization remain uncertain. There are some hints for instance that the surface of amorphous $\mathrm{SiO}_{2}$ is able to form very specific but flexible hydrogen-bonded adducts with some biomolecules, a promising feature if we remember the role played by $\mathrm{H}$-bonds in modern biochemistry. Characterizing H-bonding on surfaces at the molecular scale lies at the current limit of precision of our experimental techniques. Progress toward this goal may seem excruciatingly slow, but the more we learn, the more we become aware that very welldefined chemistry can take place on the surface of even something as mundane as amorphous silica: a recent review dedicated to modeling and experimental studies of small biomolecules adsorption on silica reached a hundred pages [58].

In addition to this tedious but rewarding progress towards molecular characterization, exploratory studies must be dedicated to the performance of surface chemistry for more complex systems than have been currently investigated. Is there some "interfacial" pathway from the disorganized complexity of the prebiotic soup towards functional systems that were the precursors of life? Only thoughtful experiments devised in cooperation with systems chemists will be able to answer this question. 


\section{ORIGINS}

\section{References}

[1] S.A. Kauffman, At Home in the Universe: The Search for Laws of Self-Organization and Complexity (Oxford University Press., Oxford, 1995)

[2] A. Pross, What is life? How chemistry becomes biology. (Oxford University Press, Oxford, 2012).

[3] P. Ehrenfreund, M.A. Sephton, Faraday Disc. 133, 277-288 (2006)

[4] P. Coll, R. Navarro-González, C. Szopa, O. Poch, S.I. Ramírez, D. Coscia, F. Raulin, M. Cabane, A. Buch, G. Israël, Planet. Space Sci. 77, 91-103 (2013)

[5] P. Ehrenfreund, W. Irvine, L. Becker, J. Blank, J.R. Brucato, L. Colangeli, S. Derenne, D. Despois, A. Dutrey, H. Fraaije, A. Lazcano, T. Owen, F. Robert, Rep. Progr. Phys. 65, 10, 1427-1487 (2002)

[6] U.J. Meierhenrich, G.M. Munoz Caro, J.H. Bredehöft, E.K. Jessberger, W.H.-P. Thiemann, Proc. Nati. Acad. Sci. USA 416, 9182-9186 (2004)

[7] G. Cooper, N. Kimmich, W. Belisle, J. Sarinana, K. Brabham, L. Garre, Nature 414, 879-883 (2001)

[8] S.L. Miller, H.C. Urey, Science 130, 3370, 245-251 (1959)

[9] R.M. Hazen, A. Bekker, D.L. Bish, W. Bleeker, R.T. Downs, J. Farquhar, J.M. Ferry, E.S. Grew, A.H. Knoll, D. Papineau, J.P. Ralph, D. Sverjensky, J.W. Valley, Amer. Mineral. 96, 953-963 (2011)

[10] H.A. Ishii, J.P. Bradley, Z.R. Dai, M.F. Chi, A.T. Kearsley, M. Burchell, N.D. Browning, F. Molster, Science 319, 5862, 447-450 (2008)

[11] H.G.M. Hill, J.A. Nuth, Astrobiol. 3, 2, 291-304 (2003)

[12] U. Meierhenrich, Amino Acids and the Asymmetry of Life - Caught in the Act of Formation (Springer Verlag, Heidelberg, 2008)

[13] R.M. Hazen, T.R. Filley, G.A. Goodfriend, Proc. Nati. Acad. Sci. USA 98, 10, 5487-5490 (2001)

[14] T. Hitz, P.L. Luisi, 86, 1423-1434 (2003)

[15] J.W. Han, D.S. Sholl, Langmuir 18, 10737-10745 (2009)

[16] E. Klabunovskii, W. Thiemann, Orig. Life Evol. Biosph. 30, 431-434 (2000)

[17] A. Brack, Chem. Biodiversity 4, 665-679 (2007)

[18] J.-F. Lambert, Orig. Life Evol. Biosph. 38, 3, 211-242 (2008)

[19] D. Bernal, The Physical basis of life (Routledge \& Kegan edn., London, 1951)

[20] N. Lahav, D. White, S. Chang, Science 201, 4350, 67-69 (1978)

[21] N. Lahav, D.H. White, J. Mol. Evol. 16, 11-21 (1980)

[22] J. Bujdák, H. Slosiariková, N. Texler, M. Schwendiger, B.M. Rode, Monatsh. Chem. 125, 10331039 (1994)

[23] J. Bujdák, B.M. Rode, React. Kinet. Catal. Lett. 62, 2, 281-286 (1997)

[24] K. Marshall-Bowman, S. Ohara, D.A. Sverjensky, R.M. Hazen, H.J. Cleaves, Geochem. Cosmochem. Acta 74, 20, 5852-5861 (2010)

[25] T. Georgelin, M. Jaber, H. Bazzi, J.-F. Lambert, Orig. Life Evol. Biosphere 43, 4-5, 429-443 (2013)

[26] J.-F. Lambert, M. Jaber, T. Georgelin, L. Stievano, Phys. Chem. Chem. Phys. 15, 32, 13371 13380 (2013)

[27] J.P. Ferris, A.R.J. Hill, R. Liu, L.E. Orgel, Nature 381, 59-61 (1996)

[28] R. Pascal, L. Boiteau, A. Commeyras, in Prebiotic chemistry: from simple amphiphiles to protocell models, pp. 69-122 (Springer Verlag, Berlin, 2005)

[29] V.A. Basiuk, T.Y. Gromovoy, Coll. Surf. A 118, 127-140 (1996)

[30] M. Jaber, T. Georgelin, H. Bazzi, F. Costa-Torro, J.-F. Lambert, G. Bolbach, G. Clodic, J. Phys. Chem. C 118, 44, 25447-25455 (2014) 
[31] J. Bujdák, K. Faybikova, A. Eder, Y. Yongyai, B.M. Rode, Orig. Life Evol. Biosph. 25, 5, 431-441 (1995)

[32] J. Bujdák, B.M. Rode, J. Inorg. Biochem. 90, 1-7 (2002)

[33] P. Leyton, R.A. Zárate, S. Fuentes, C. Paipa, J.S. Gómez-Jeria, Y. Leytond, Biosystems 104, 118-126 (2011)

[34] P. Leyton, R. Saladino, C. Crestini, M. Campos-Vallette, C. Paipa, A. Berrios, S. Fuentes, R.A. Zarate, Amino Acids 42, 6, 2079-2088 (2012)

[35] J. Ren, L. Xin, Y.-N. Liu, K.-J. Wang, Macromol. 41, 1996-2002 (2008)

[36] G. Ertem, J.P. Ferris, Orig. Life Evol. Biosph. 30, 411-422 (2000)

[37] G. Martra, C. Deiana, Y. Sakhno, I. Barberis, M. Fabbiani, M. Pazzi, M. Vincenti, Angew. Chem. 53, $4671-4674$ (2014)

[38] G. Wächtershäuser, Microbiol. Rev., 452 (1988)

[39] G. Wächtershäuser, System. AppI. MicrobioI. 10, 207-210 (1988)

[40] G. Wächtershäuser, Proc. Nati. Acad. Sci. USA 87, 200-204 (1990)

[41] G. Wächtershäuser, Progr. Biophys. Molec. Biol. 58, 85-201 (1992)

[42] C. Huber, G. Wächtershäuser, Science 276, 5310, 245-247 (1997)

[43] C. Huber, G. Wächtershäuser, Science 281, 670-672 (1998)

[44] L.M. Barge, I.J. Doloboff, M.J. Russell, D. VanderVelde, L.M. White, G.D. Stucky, M.M. Baum, J. Zeytounian, R. Kidd, I. Kanik, Geochim. Cosmochim. Acta 128, 1-12 (2014)

[45] A.G. Cairns-Smith, J. Theoret. Biol. 10, 53-88 (1966)

[46] A.G. Cairns-Smith, Genetic Takeover and the Mineral Origins of Life (Cambridge University Press, Cambridge, 1982)

[47] A.G. Cairns-Smith, Seven Clues to the Origin of Life: A Scientific Detective Story (Cambridge University Press, Cambridge, 1985)

[48] T. Bullard, J. Freudenthal, S. Avagyan, B. Kahr, Faraday Disc. 136, 231-245 (2007)

[49] D. Schumann, H. Hartman, D.D. Eberl, S.K. Sears, R. Hesse, H. Vali, Astrobiol. 12, 6, 549-561 (2012)

[50] J.L. Cape, P.-A. Monnard, J.M. Boncella, Chem. Sci. 2, 661-671 (2011)

[51] D.W. Deamer, R.M. Pashley, Orig. Life Evol. Biosph. 19, 1, 21-38 (1989)

[52] J.V. Smith, Proc. Nati. Acad. Sci. USA 95, 3370-3375 (1998)

[53] J.V. Smith, F.P. Arnold Jr, I. Parsons, M.R. Lee, Proc. Nati. Acad. Sci. USA 96, 3479-3485 (1999)

[54] M.D. Brasier, R. Matthewman, S. McMahon, D. Wacey, Astrobiology 11, 7, 725-735 (2011)

[55] H.G. Hansma, J. Theoret. Biol. 266, 165-188 (2010)

[56] C. de Duve, S.L. Miller, Proc. Natl. Acad. Sci. USA 88, 10014-10017 (1991)

[57] M.M. Hanczyc, S.M. Fujikawa, J.W. Szostak, Science 302, 618-622 (2003)

[58] A. Rimola, D. Costa, M. Sodupe, J.-F. Lambert, P. Ugliengo, Chem. Rev. 113, 4216-4313 (2013) 